University of Wollongong

Research Online

Faculty of Informatics - Papers (Archive)

Faculty of Engineering and Information

Sciences

17-10-2007

\title{
Diversity Performance of Interleaved Spread Spectrum OFDM Signals over Frequency Selective Multipath Fading Channels
}

P. Tu

University of Wollongong

Xiaojing Huang

University of Wollongong, huang@uow.edu.au

E. Dutkiewicz

University of Wollongong, eryk@uow.edu.au

Follow this and additional works at: https://ro.uow.edu.au/infopapers

Part of the Physical Sciences and Mathematics Commons

\section{Recommended Citation}

Tu, P.; Huang, Xiaojing; and Dutkiewicz, E.: Diversity Performance of Interleaved Spread Spectrum OFDM Signals over Frequency Selective Multipath Fading Channels 2007.

https://ro.uow.edu.au/infopapers/641

Research Online is the open access institutional repository for the University of Wollongong. For further information contact the UOW Library: research-pubs@uow.edu.au 


\title{
Diversity Performance of Interleaved Spread Spectrum OFDM Signals over Frequency Selective Multipath Fading Channels
}

\author{
Abstract \\ In this paper we propose an interleaved spread spectrum orthogonal frequency division multiplexing (ISS- \\ OFDM) system for achieving both frequency diversity and time diversity and investigate the system \\ performance over frequency selective fading channels. The purpose is to exploit the diversity capability of \\ OFDM systems, develop efficienct spectrum spreading technique and improve the system performance \\ against frequency selective channel fading. At the transmitter, the ISS-OFDM signal is generated by \\ employing spread spectrum modulation and interleaving techniques. At the receiver, the received signals \\ are combined by using maximum ratio combining (MRC) technique. The simulation indicates that the ISS- \\ OFDM system has improved performance in multipath fading channels. Another unique characteristic is \\ that the spectrum spreading factor and diversity order provided by the system are reconfigurable to \\ achieve cognitive communications.

\section{Disciplines} \\ Physical Sciences and Mathematics

\section{Publication Details} \\ This conference paper was originally published as Tu, P, Huang, X, Dutkiewicz, E, Diversity Performance of \\ Interleaved Spread Spectrum OFDM Signals over Frequency Selective Multipath Fading Channels, \\ International Symposium on Communications and Information Technologies ISCIT 2007, 17-19 Oct, \\ 184-189.
}




\title{
Diversity Performance of Interleaved Spread Spectrum OFDM Signals over Frequency Selective Multipath Fading Channels
}

\author{
Pingzhou Tu, Xiaojing Huang, Eryk Dutkiewicz \\ Email: \{pt015, huang, eryk\}@uow.edu.au \\ School of Electrical, Computer and Telecommunications Engineering \\ University of Wollongong, Australia
}

\begin{abstract}
In this paper we propose an interleaved spread spectrum orthogonal frequency division multiplexing (ISS-OFDM) system for achieving both frequency diversity and time diversity and investigate the system performance over frequency selective fading channels. The purpose is to exploit the diversity capability of OFDM systems, develop efficienct spectrum spreading technique and improve the system performance against frequency selective channel fading. At the transmitter, the ISS-OFDM signal is generated by employing spread spectrum modulation and interleaving techniques. At the receiver, the received signals are combined by using maximum ratio combining (MRC) technique. The simulation indicates that the ISS-OFDM system has improved performance in multipath fading channels. Another unique characteristic is that the spectrum spreading factor and diversity order provided by the system are reconfigurable to achieve cognitive communications.
\end{abstract}

\section{INTRODUCTION}

Frequency selective fading is a dominant impairment in wireless communications. The channel fading reduces received signal-to-noise ratio (SNR) and degrades the bit error rate (BER). It also causes channel delay spread and introduces inter-symbol interferences (ISI) [1]. To combat the frequency selective fading in wireless communications, diversity techniques must be resilient [2]. Orthogonal frequency division multiplexing (OFDM) technique as one of the multi-carrier transmission techniques has extended the symbol duration and effectively reduced the ISI by inserting cyclic prefix, which is greater than the channel delays, before the signal is transmitted to the channel. OFDM has been considered in a wide range of applications in recent years, but its diversity potentials have not been fully exploited yet.

Driven by OFDM applications against channel fading and by enabling multipath diversity, some different forms of OFDM systems with different diversity techniques have been proposed, such as the multiple-input multiple-output OFDM (MIMO-OFDM) systems [3], the frequency diversity OFDM (FD-OFDM or AFD-OFDM) systems [4], and the spreadspectrum multiple-carrier multiple-access (SS-MC-MA) [5]. Among these systems, the MIMO-OFDM is one of the most typical ones. It adopts a very commonly used method for achieving spatial diversity by employing multiple antennas at the both ends of wireless links. It holds the potential to drastically improve the spectral efficiency and link reliability in future wireless communications systems, and is regarded as a particularly promising candidate for next-generation fixed and mobile wireless systems. However, an open issue for MIMOOFDM systems is on realizing that promising technique in real time [6]. The other two methods, FD-OFDM and SSMC-MA, use the same way of multiplying the transmitted data stream by an orthogonal spreading code before modulation in order to achieve frequency diversity. The spectrum spreading is accomplished by putting the same data on all parallel subcarriers, producing a spreading factor equal to the number of subcarriers. Although these systems enable full multipath diversity, they need a large number of side information transmission, which causes considerable rate loss $[7,8]$.

In this paper we propose a method for achieving both time diversity and frequency diversity and the corresponding receiver algorithm for diversity combining. The proposed system is called interleaved spread spectrum orthogonal frequency division multiplexing (ISS-OFDM) system. In this system, both time diversity and frequency diversity are enabled by a spread spectrum modulation and interleaving technique, rather than using multiple antennas, orthogonal codes, or other side information. At the transmitter, each data symbol modulates the corresponding subcarriers multiple times, and several replicas bearing the same data information can be obtained. The replicas of the same data information are interleaved into serial sequence and then transmitted in different time slots, instead of being added together. Thus, time diversity is achieved since the replicas of a data symbol are placed in different time slots. The interleaving operation also results in signal spectrum being expanded into multiple subbands, each of which contains the same data information, so that frequency diversity is achieved. After frequency selective channel fading, the receiver can collect signal energy from several signal subbands by using maximal ratio combining (MRC) techniques. Thus, the probability that all signal components fade simultaneously is reduced considerably, leading to great system performance improvement.

The paper is organized as follows. First, the system model is described in Section II. Then, the OFDM signal generation process with both time diversity and frequency diversity is described in Section III, where the signal diversity characteristics are analyzed in frequency domain and time domain. In 
Sections IV, the corresponding receiver models are proposed. Section V is devoted to system performance simulation and discussion, in which the system average bit error probability and computational complexity are given. Finally, conclusions are drawn in Section VI.

\section{SYSTEM MODEL}

Fig. 1 displays the ISS-OFDM baseband system model. We assume that the data bit to symbol mapping scheme is quadrature phase shift keying (QPSK), the channel is frequency selective with slow fading, and the noise is additive white Gaussian noise (AWGN) with zero mean. A cyclic prefix $(\mathrm{CP})$ is also added to preserve the orthogonality of the subcarriers and eliminate intersymbol interference (ISI) between consecutive ISS-OFDM symbols.

Under the above assumptions we now describe the function of each module showed in Fig. 1. The function of the transmitter is to generate an ISS-OFDM signal with time and frequency diversities. The complex QPSK data symbols to be transmitted are first divided into an $N$ by 1 vector, where $N$ is the number of subcarriers. This vector modulate $N$ subcarries in parallel by using a spread spectrum modulation technique, which we called as complex exponential spreading (CES), and then the spread signals are interleaved to form an ISS-OFDM symbol of $N^{2}$ samples. After cyclic prefix insertion and filtering, the ISS-OFDM signal $y(m)$ (also represented by $Y(k)$ in the frequency domain) with spectrum spreading is transmitted into the frequency selective multipath fading channel with impulse response $h(m)$ (denoted as $H(k)$ in the frequency response ). Meanwhile, the AWGN noise $v(m)$ (denoted as $V(k)$ in the frequency domain) is added to the signal. After distorted by the fading channel, the signal is filtered and CP removed, and then demodulated. The output of the demodulator is equalized and combined to form a decision variable $U_{k}$ by employing maximal ratio combining (MRC) technique.

As we all know, when signals are propagated in a frequency selective multipath fading channel, the presence of reflecting objects and scatters in the channel creates a constantly changing environment that dissipates the signal energy in amplitude, phases and time. These effects result in multiple versions of the transmitted signal, which are received by the receiver at different times from different multipaths, displaced with respect to one another in time and spatial orientation. We may assume that the transmitted ISS-OFDM signal bandwidth $B_{I S S-O F D M}$ is greater than the coherence bandwidth $(\triangle f)_{c}$, $B_{I S S-O F D M} \gg(\triangle f)_{c}$ thus, the channel can be considered as frequency-selective fading channel. The channel model can be expressed in the form

$$
h(m)=\sum_{i=0}^{N^{2}-1} \alpha_{i}(m) \delta\left(m-m_{i}\right)
$$

where $\alpha_{i}(m)$ is the multipath fading channel attenuation factor on the $m^{t h}$ path and $m_{i}$ is the propagation delay for the

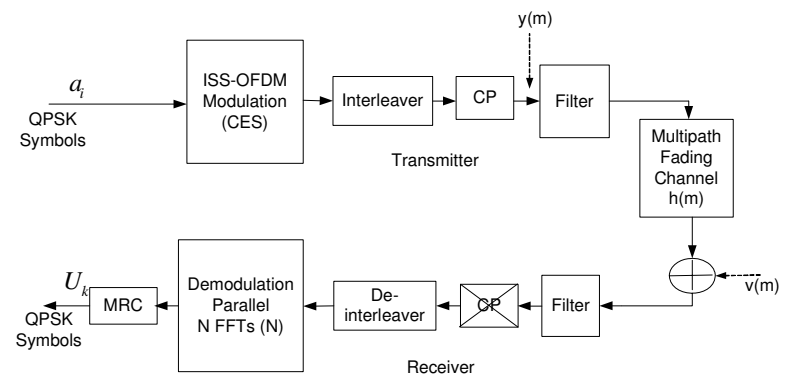

Fig. 1. Baseband system model.

$m^{t h}$ path. Correspondingly, the channel frequency response is written as

$$
H(k)=\sum_{m=0}^{N^{2}-1} h(m) e^{-j \frac{2 \pi}{N^{2}} k m} .
$$

After the transmitted signal, denoted as $y(m)$, is passed through the fading channel, the received equivalent low-pass signal is viewed as the convolution between $h(m)$ and $y(m)$ plus noise as follows

$$
r(m)=h(m) \otimes y(m)+v(m)
$$

where $v(m)$ represents the complex-valued white Gaussian noise process corrupting the signal. In frequency domain Equation (2) is equivalent to the output of FFT demodulator,

$$
R(k)=H(k) \cdot Y(k)+V(k)
$$

where $R(k), Y(k), H(k), V(k)$ denote the frequency representations of the received signal $r(m)$, the transmitted signal $y(m)$, the channel impulse response $h(m)$, and the noise $v(m)$, respectively.

\section{DIVERSITY IN ISS-OFDM SIGNALS}

\section{A. Transmitter Model}

Fig. 2 shows the transmitter model, which indicates the generation process of ISS-OFDM signal with the time diversity and frequency diversity. Let $a_{i}(i=0,1, \cdots, N)$ denote the $i^{\text {th }}$ complex-valued symbol of the NQPSK symbols required to be transmitted. The $N$ QPSK symbols are modulated by the CES modulator, and then interleaved by the interleaver to form ISS-OFDM signal with time diversity and frequency diversity. Time diversity is achieved by CES modulation and frequency diversity is obtained by interleaving.

\section{B. Time Diversity of ISS-OFDM system}

After serial to parallel converter the $N \times 1$ parallel QPSK symbols modulate the corresponding $N$ subcarriers. If the ISSOFDM symbol period is $T_{s}, f_{i}=\frac{i}{T_{s}}$ denotes the $i^{t h}$ subcarrier frequency of the $N$ orthogonal subcarriers, and $a_{i}$ modulates the $i^{t h}$ subcarrier at time $t=\frac{n}{N} T_{s}, n=0,1, \cdots, N-1$, where $n$ is the sampling time index. The modulated symbol on the $i^{\text {th }}$ subcarrier and the $n^{\text {th }}$ time instant is written as follows, 


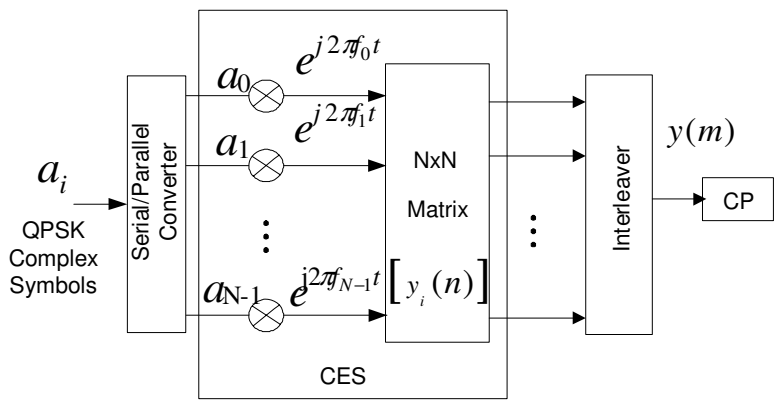

Fig. 2. Transmitter model.
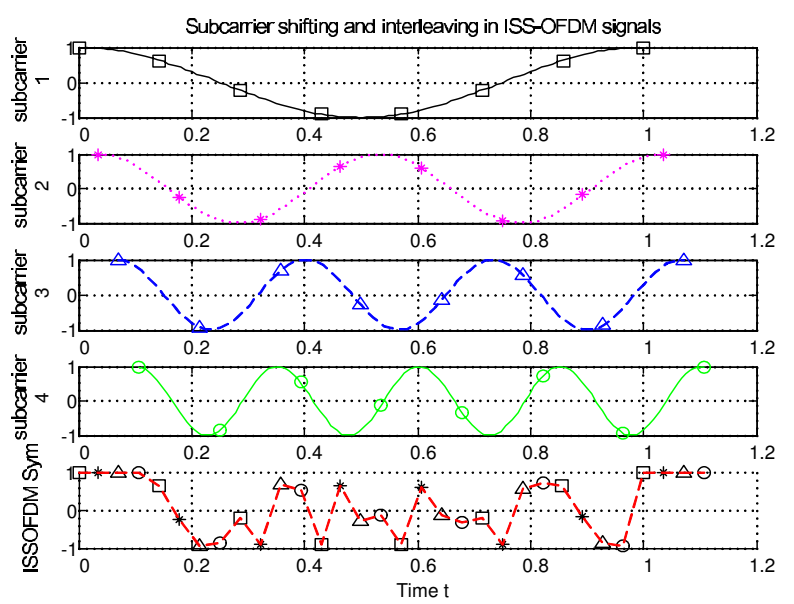

Fig. 3. Time diversity of ISS-OFDM signals with subcarrier number $\mathrm{N}=4$.

$$
y_{i}(n)=a_{i} e^{j 2 \pi f_{i} t}=a_{i} e^{j 2 \pi n i / N} .
$$

In ISS-OFDM symbol duration $T_{s}$, each element of the $N \times 1$ data symbol vector modulates the same corresponding subcarrier $N$ times, so that $N$ elements in the vector generate an $N \times N$ matrix after modulation. In consequence, $N$ replicas of each data symbol are produced in symbol duration $T_{s}$ by CES modulation, and the time diversity of the ISS-OFDM symbol is achieved. Fig. 3 displays the process of achieving time diversity in the ISS-OFDM system.

\section{Frequency Diversity of ISS-OFDM Signals}

After CES modulation, the $N \times N$ samples are shifted in time and placed on different time slots to form a serial sequence of length $N^{2}$ in the time domain. This operation is a process of interleaving. Here, different interleaving algorithms can be employed such as pseudo random interleaving, periodic interleaving and convolutional interleaving. In the following, we discuss the periodic interleaving process. The periodic interleaving can be realized by shifting $N$ modulated subcarriers on a different time slots and adding them together. For instance, the $i^{t h}$ subcarrier of the $N$ subcarriers is shifted by $i \cdot \tau$, where $\tau$ is the sampling interval, $\tau=\frac{T_{s}}{N^{2}}$. Then, the $N$ shifted subcarriers are added together to form one ISS-OFDM symbol with $N^{2}$ samples. The $m^{\text {th }}$ sample in the ISS-OFDM symbol, denoted by $y(m), m=n N+i=0,1, \cdots, N^{2}-1$, can be mathematically written as follows,

$$
y(m)=\sum_{i=1}^{N} \sum_{n}^{N} y_{i}(n) \delta[m-i-n N]
$$

where $\delta[m-n N-i]=\left\{\begin{array}{l}1, \text { for } m=n N+i \\ 0, \text { for } m \neq n N+i .\end{array}\right.$ is the unit impulse. Assuming $m=n N+i, y(m)$ can be simplified as the form

$$
y(m)=y(n N+i)=y_{i}(n) \quad n, i=0,1, \cdots, N-1 .
$$

The mathematical equations above can be interpreted as follows. All $N$ samples in a column of the $N \times N$ matrix are taken out from the $N \times N$ matrix, shifted in time, and then placed in different time slots, instead of being superimposed together as in conventional OFDM systems. Due to the shifting in time for each sample, the phase of each subcarrier is shifted. In frequency domain, the signal spectrum is expanded to $N$ subbands, each of which contains $N$ orthogonal subcarriers modulated by the same transmitted data symbols. Therefore, the ISS-OFDM signal's frequency diversity is obtained.

Fig. 4 displays the spectrum of an ISS-OFDM signal with $N=4$ subcarriers. It is seen that the modulated data symbol on the $i^{\text {th }}$ (in case of $\left.i=1,2,3,4\right)$ subcarrier appears in all $N$ subbands.

Mathematically, the frequency domain representation of the ISS-OFDM signal with frequency diversity can be obtained by performing FFT operation on signal in time domain.

$$
\begin{aligned}
& Y(k)=F F T(y(m))=F F T(y(n N+i)) \\
& =\sum_{n N+i=0}^{N^{2}-1} y(n N+i) e^{-j 2 \pi N^{2}(n N+i) k} \\
& Y(k)=\sum_{n=0}^{N-1} \sum_{i=0}^{N-1} y(n N+i) e^{-j \frac{2 \pi}{N^{2}}(n N+i) k} .
\end{aligned}
$$

Substituting $y(n N+i)$ from Equation (5) into Equation (6),we have

$$
Y(k)=\sum_{n=0}^{N-1} \sum_{i=0}^{N-1} y_{i}(n) e^{-j \frac{2 \pi}{N^{2}}(n N+i) k} .
$$

Substituting $y_{i}(n)$ from Equation (4) into Equation (7) yields

$$
\begin{gathered}
Y(k)=\sum_{n=0}^{N-1} \sum_{i=0}^{N-1} a_{i} e^{j \frac{2 \pi}{N} n i} e^{-j \frac{2 \pi}{N^{2}}(n N+i) k} \\
=N \sum_{i=0}^{N-1} a_{i} e^{-j \frac{2 \pi}{N^{2}} k i} \underbrace{\frac{1}{N} \sum_{n=0}^{N-1} e^{-j \frac{2 \pi}{N}(k-i) n}}_{\delta\left((k-i)_{N}\right)} \\
Y(k)=N \sum_{i=0}^{N-1} a_{i} e^{-j \frac{2 \pi}{N^{2}} k i} \delta\left((k-i)_{N}\right)
\end{gathered}
$$




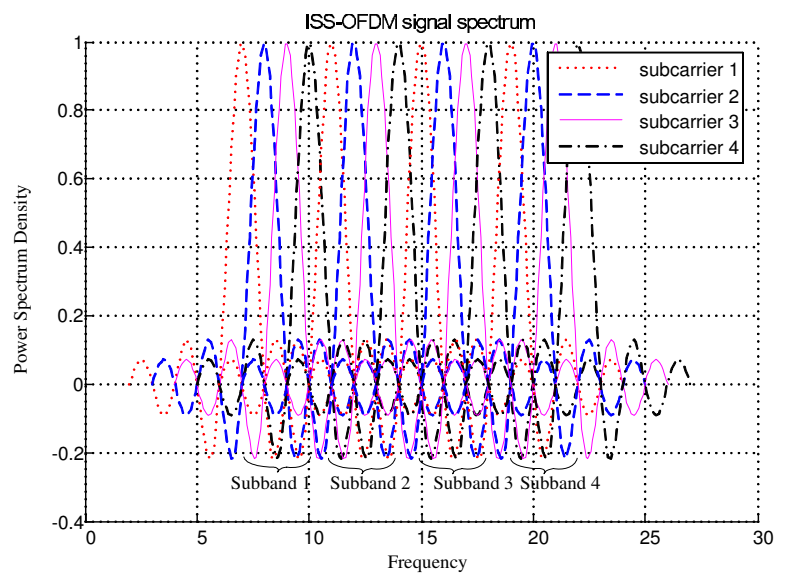

Fig. 4. Spectrum of ISS-OFDM symbol with subcarrier number $\mathrm{N}=4$.

$$
\begin{gathered}
Y(p N+i)=Y_{p}(i) \\
=N a_{i} e^{-j \frac{2 \pi}{N^{2}}(p N+i) i} \text {, for } m=n N+i
\end{gathered}
$$

where $i, p=0,1, \cdots, N-1$.

Equation (9) indicates that each data symbol $a_{i}$ is modulated on the $i$ subcarrier in every subband, and the signal spectrum is spread $N$ times. Fig. 4 shows the spectrum of the ISS-OFDM signal with subcarriers number $N=4$.

\section{DIVERSITY COMBINING OF ISS-OFDM SIGNALS}

At the receiver, the received signal is firstly filtered by using a root raised cosine filter. The filter is matched to the transmitter filter, so that the overall effect of filtering is that of a raised cosine filtering. The passband of the receiver filter is designed to be flexibly reconfigurable according to the system requirements. One or more subbands of received signal can be selected through receiver filter. Assuming that all the subbands of the received signal are passed through the filter, the diversity combining can be conducted as follows.

\section{A. Frequency Domain Representation of Received Signal}

Substitute $Y(k)$ from Equation (8) into Equation (3), the received diversity signal at the input of the receiver in frequency domain is rewritten as follows

$$
R(k)=N \sum_{i=0}^{N-1} a_{i} \underbrace{\delta\left((k-i)_{N}\right)}_{k=p N+i} e^{-j \frac{2 \pi}{N^{2}} k i} H(k)+V(k)
$$

where $k=0,1, \cdots, N^{2}-1$,

$$
R(k)=N \sum_{i=0}^{N-1} a_{i} \underbrace{H(p N+i) e^{-j \frac{2 \pi}{N^{2}}(p N+i) i}}_{C_{i, k}}+V(k)
$$

where $C_{i, k}$ denotes $H(p N+i) e^{-j \frac{2 \pi}{N^{2}}(p N+i) i}$, and $p=$ $0,1, \cdots, N$. That is,

$$
R(k)=N \sum_{i=0}^{N} a_{i} \cdot C_{i, k}+V(k) .
$$

\section{B. Parallel Demodulation by FFT}

Fig. 5 displays the receiver model for demodulation and diversity combining, which is realized by using $N$ parallel FFTs, each of which is of size $N$. To obtain the output of each FFT operation, we begin with the received signal in time domain and split the signal into $N$ groups, each of which is demodulated by a FFT operation of size $N$. From Equation (10) and (11), the time domain received signal at the input of the receiver can be alternatively expressed after performing the IFFT operation of size $N^{2}$ on $R(k)$, which is written as

$$
\begin{aligned}
& r(m)=\operatorname{IFFT}(R(k))=\frac{1}{N^{2}} \sum_{k=0}^{N^{2}-1} R(k) e^{j \frac{2 \pi}{N^{2}} k m} \\
& =\frac{1}{N} \sum_{k=0}^{N^{2}-1} \sum_{i=0}^{N-1} a_{i} e^{-j \frac{2 \pi}{N^{2}} k(i-m)} \delta\left((i-k)_{N}\right) H(k) \\
& +\frac{1}{N^{2}} \sum_{k=0}^{N^{2}-1} V(k) e^{j \frac{2 \pi}{N^{2}} k m}
\end{aligned}
$$

The received signal $r(m)$ can be deinterleaved into $N$ streams. Each stream $r_{i}(n), i=0,1, \cdots, N-1$ can be expressed as

$$
r_{i}(n)=r(n N+i)=r(m)
$$

where $\quad i=0,1, \cdots N-1 ., \quad n=0,1, \cdots, N-1$.

$$
\begin{gathered}
R_{i}(k)=\sum_{n=0}^{N-1} r_{i}(n) e^{-j \frac{2 \pi}{N} k n} \\
=a_{k} \underbrace{\sum_{q=0}^{N-1} e^{j \frac{2 \pi}{N} q(i-k)} H(q N+k) e^{-j \frac{2 \pi}{N^{2}} i(k-i)}}_{\theta_{i, k}} \\
+\underbrace{\frac{1}{N} \sum_{q=0}^{N-1} V(q N+k) e^{j \frac{2 \pi}{N^{2}}(q N+k) i}}_{\nu_{i, k}}
\end{gathered}
$$

where $k=0,1, \ldots, N-1$,

$$
\theta_{i, k}=\sum_{q=0}^{N-1} e^{j \frac{2 \pi}{N} q(i-k)} H(q N+k) e^{-j \frac{2 \pi}{N^{2}} i(k-i)}
$$

and $\nu_{i, k}=\frac{1}{N} \sum_{q=0}^{N-1} V(q N+k) e^{j \frac{2 \pi}{N^{2}}(q N+k) i}$.

Equation (13) indicates that the output signal on each stream of demodulator equals the corresponding transmitted signal $a_{k}$ multiplied channel attenuation factor $\theta_{i, k}$. Substituting $\theta_{i, k}$, and $\nu_{i, k}$ into Equation (13), we obtain the simplified expressions,

$$
R_{i}(k)=a_{k} \cdot \theta_{i, k}+\nu_{i, k} .
$$




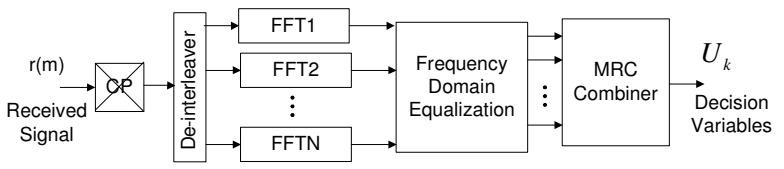

Fig. 5. Parallel demodulation and combination.

\section{Diversity Combining}

Before diversity combining is performed, the demodulated signals must be equalized. Referring to Equation (14), the equalized signal is

$$
\hat{R_{i}(k)}=\left(\theta_{i, k}\right)^{*} R_{i}(k)=\left(\theta_{i, k}\right)^{*} \cdot \theta_{i, k} a_{k}+\left(\theta_{i, k}\right)^{*} \nu_{i, k} .
$$

Since $\theta_{i, k}$ are mutually statistically independent, MRC combination is used to generate decision variable $U_{k}$,

$$
\begin{gathered}
U_{k}=\left[\sum_{i=0}^{N-1}\left(\theta_{i, k}\right)^{*} \cdot \theta_{i, k} a_{k}\right]+\left[\sum_{i=0}^{N-1}\left(\theta_{i, k}\right)^{*} \nu_{i, k}\right] \\
U_{k}=\sum_{i=0}^{N-1}\left|\theta_{i, k}\right|^{2} a_{k}+\sum_{i=0}^{N-1}\left(\theta_{i, k}\right)^{*} \nu_{i, k} .
\end{gathered}
$$

\section{Diversity Performance}

For a fixed set of $\left\{\theta_{i, k}\right\}$, the decision variable $U_{k}$ is Gaussian with mean

$$
E\left[U_{k}\right]=E\left[\sum_{i=0}^{N-1}\left|\theta_{i, k}\right|^{2} a_{k}+\sum_{n=0}^{N-1}\left(\theta_{i, k}\right)^{*} \nu_{i, k}\right] .
$$

Since $\left\{\theta_{i, k}\right\}$ is fixed and $\nu_{i, k}$ is Gaussian distribution with mean equals to zero, $E\left(\sum_{n=0}^{N-1}\left(\theta_{i, k}\right)^{*} \nu_{i, k}\right)=0$, thus, the mean of $U_{k}$ can be simplified as

$$
E\left[U_{k}\right]=E\left(\sum_{i=0}^{N-1}\left|\theta_{i, k}\right|^{2} a_{k}\right)=\sum_{i=0}^{N-1}\left|\theta_{i, k}\right|^{2} \underbrace{a_{k}}_{\sigma_{y}}
$$

and variance $\sigma_{U_{k}}^{2}=\sum_{i=0}^{N-1}\left|\theta_{i, k}\right|^{2} \underbrace{\nu_{i, k}^{2}}_{\sigma_{v}^{2}}=\sum_{i=0}^{N-1}\left|\theta_{i, k}\right|^{2} \sigma_{v}^{2}$ where $\sigma_{y}^{2}$ and $\sigma_{v}^{2}$ denote signal power and noise power per sample respectively.

For these values of the mean and variance, the probability that $U_{k}$ is less than zero (assuming $a_{k}>0$ ), i.e., bit error rate (BER), is simply

$$
P_{U}=Q(\sqrt{\gamma})
$$

where the signal to noise ratio (SNR) per sample, is given as

$$
\begin{aligned}
& \gamma=\frac{\left(E\left(U_{k}\right)\right)^{2}}{\sigma_{U}^{2}}=\frac{\left(\sum_{i=0}^{N-1}\left|\theta_{i, k}\right|^{2}\right)^{2} \sigma_{y}^{2}}{\sum_{i=0}^{N-1}\left|\theta_{i, k}\right|^{2} \sigma_{v}^{2}}=\sum_{i=0}^{N-1}\left|\theta_{i, k}\right|^{2} \frac{\sigma_{y}^{2}}{\sigma_{v}^{2}} \\
& =\sum_{i=0}^{N-1}\left|\theta_{i, k}\right|^{2} \cdot S N R .
\end{aligned}
$$

Denoting $C=\sum_{i=0}^{N-1}\left|\theta_{i, k}\right|^{2}, P_{U}$ can be simplified as

$$
P_{U}=Q(\sqrt{C \cdot S N R}) .
$$

The final step in this derivation is to statistically average the conditional error probability $P_{U}$ given in Equation (15) over the fading variable $C=\sum_{i=0}^{N-1}\left|\theta_{i, k}\right|^{2}$. Thus, we evaluate the expectation of $P_{U}$ as

$$
P_{e}=E_{C}\left(P_{U}\right)=E_{C}(Q(\sqrt{C \cdot S N R}))
$$

where $E_{C}(\cdot)$ denotes the expectation of the conditional error probability over the fading channel statistics $C$.

\section{SYSTEM PERFORMANCE}

\section{A. Scalability of Diversity}

A distinct feature in the ISS-OFDM system is the scalability of the signal diversities. Since the signal spectrum is very wide and contains $N$ subbabds, each of which contains the same data information, any one or more subbands can be used to demodulate the transmitted information according to the system requirement and channel condition. At the transmitter, if we configure the transmitter filter so that the signal spectrum is spread $M$ times, $M \leqslant L$, where $L$ is the length of multipath channels, the system frequency diversity order would be $M$. The diversity order can be achieved dynamically by adjusting the parameters of modulation and filters, and the diversity capability of the ISS-OFDM system is dependent on the number of subbands or spreading factor. If the number of the subcarriers of the ISS-OFDM is $N$, then the maximum spectrum spreading factor is $N$ and the maximum diversity capability of ISS-OFDM system is $N$. The more the diversity capability is provided by the system, the better BER performance the system can achieve. However, if the diversity capability provided by the system is more than the channel multipath $L$, the extra diversity capability provided by the system is redundant. That is to say, if $M>L$ the diversity of the ISS-OFDM system is limited to the channel multipath length $L$.

The proposed ISS-OFDM can be used to achieve cognitive communications with dynamic subband allocation. If some of the subbands are interfered by other systems, the ISSOFDM system can jump to other not-interfered subbands to complete communications and effectively avoid interference. As indicated in Fig. 6, the system can realize communications by avoiding interference bands or by using some spectrum holes.

\section{B. BER Performance}

In Equation (16), we derived the BER performance of the ISS-OFDM system, when the ISS-OFDM transmitted signal $y(m)$ contains $N$ subbands. The system scalability can be developed by reducing the $N$ subbands of the transmitted signal to $M$ subbands if $M \leq N$. If the transmitted signal $y(m)$ contains $M$ subbands of the transmitted bandwidth, each containing $N$ subcarriers, then the total number of samples is $M \times N$. We assume the signal power per sample is $\sigma_{y}^{2}$ and the 


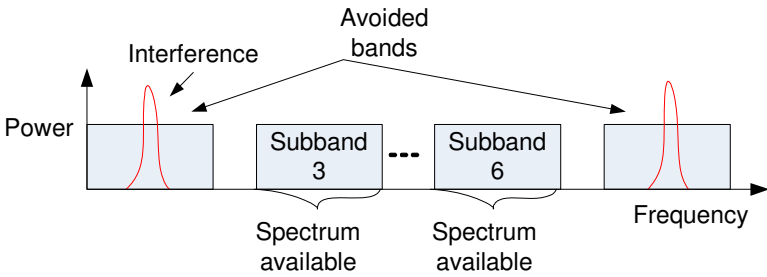

Fig. 6. Bandwidth reconfigurable system and efficient spectrum usage.

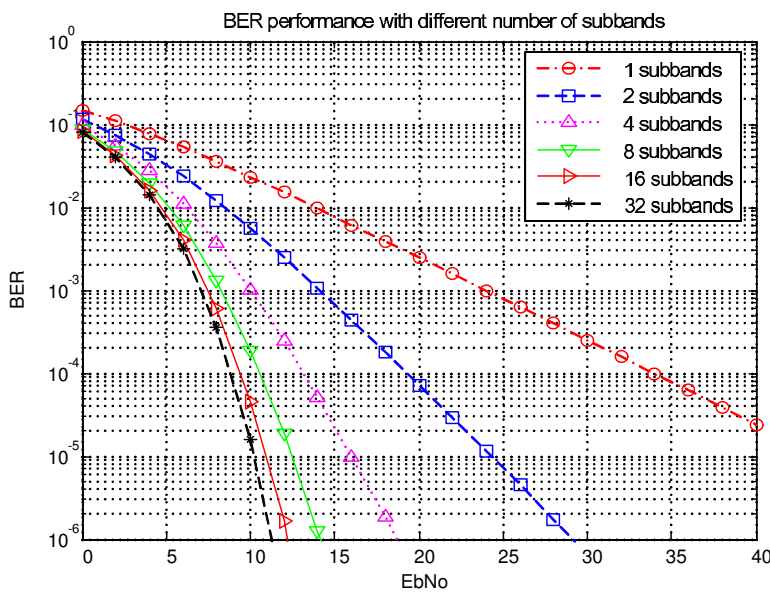

Fig. 7. BER performance of the system.

noise power is $\sigma_{v}^{2}$. Then, the relationship between the signal to noise ratio (SNR) and the ratio of bit energy (Eb) to noise power spectral density (No) can be found as follows,

$$
S N R=\frac{\sigma_{x}^{2}}{\sigma_{v}^{2}}=\frac{2}{M} \frac{E_{b}}{N_{0}} .
$$

Substituting SNR in Equation (17) into Equation (15), the bit error probability can be expressed by Q function as follows,

$$
P_{e}=E_{C}\left(Q\left(\sqrt{C \cdot \frac{2}{M} \frac{E_{b}}{N_{0}}}\right)\right) .
$$

Fig. 7 displays the BER performance of the ISS-OFDM system simulated according to Equation (18). We obtain system BER performance of the ISS-OFDM system with the number $M$ equal to $1,2,4,8,16$ and 32 respectively. It is seen that with the number of subbands increasing, the diversity increases and the system BER performance is improved distinctively. When the number of subbands increases to the number of subcarriers, $M=N$, the lower bound of the diversity performance is reached.

\section{CONCLUSION}

In this paper, we have exploited the time diversity and frequency diversity of the ISS-OFDM system and investigated its BER performance. The investigation indicates that, by exploiting the diversity provided by the ISS-OFDM system, the performance in terms of BER is improved effectively. The combination of spread spectrum modulation and interleaving techniques is an efficient way of achieving frequency diversity and implementing spread spectrum communications. Furthermore, compared with other previous spread spectrum OFDM systems, ISS-OFDM signal is superior in terms of BER, peakto-average power ratio, reconfigurable system structure, and flexible system bandwidth usage. The proposed method of achieving diversity in ISS-OFDM system can be used in ultrawide band communications. It is also expected to be used in cognitive radio adaptive modulation techniques [9].

\section{REFERENCES}

[1] J. G. Proakis, Digital Communications, 4 ed. New York, USA: Mc Graw Hill, 2000.

[2] R. Novak and W. A. Krzymien, "Diversity Combining Options for Spread Spectrum OFDM in Frequency Selective Channels," Wireless Communications and Networking Conference, vol. 1, pp. 308-314, 2005.

[3] R. S. Blum, Y. Li, J. H. Winters and Q. Yan, "Improved Space-Time Coding for MIMO-OFDM Wireless Communications," IEEE Transactions on Communications, vol. 49, no. 11, pp. 1873-1878, 2001.

[4] S. W. Kim, K. H. Yoon, R. G. Jung, J. W. Son and H. G. Ryu, "Adaptive Frequency Diversity OFDM (AFD-OFDM) Communication Narrow-Band," oint Conference of 10th Asia-Pacific Conference on Communications and 5th International Symposium on Multi-Dimensional Mobile Communicaitons, vol. 1 and 2, pp. 834-838, 2004.

[5] S. Kaiser and K. Fazel, "A Flexible Spread-Spectrum Multicarrier Multiple-Access System for Multi-Media Applications,” Personal, Indoor and Mobile Radio Communications, vol. 1, pp. 100-104, 1997.

[6] V. Jungnickel, T. Haustein, A. Forck, S. Schiffermueller, H. Gaebler and C. von Helmolt, "Real-Time Concepts for MIMO-OFDM," Proceedings CIC/IEEE Global Mobile Congress,Shanghai,China,11-13 October 2004.

[7] G. J. Saulnier, Z. Ye and M. J. Medley, "Performance of a Spread Spectrum OFDM System in a Dispersive Fading Channel with Interference," Military Communications Conference, vol. 2, pp. 679-683, 1998.

[8] P. Xia, S. Zhou and G. B. Giannakis, "Bandwidth- and Power-Efficient Multicarrier Multiple Access," IEEE Transations on Communications, vol. 51, no. 11, pp. 1828-1836, 2003.

[9] S. Haykin, "Cognitive Radio:Brain-Empowered Wireless Communications," IEEE Journal on Selected Areas in Communications, vol. 23, no. 2, pp. 201-219, 2005. 